\title{
ON A QUESTION OF RAMACHANDRA
}

\author{
By Hugh L MONTGOMERY*
}

Let $a_{k}(n)$ be the Dirichlet series coefficients defined by the relation

$$
\left(\sum_{p} \frac{1}{p^{s}}\right)^{k}=\sum_{n=1}^{\infty} \frac{a_{k}(0)}{n^{s}} \quad(\operatorname{Res}>1) .
$$

Here $k$ is a positive loteger, and we see more explicitly that

$$
\text { if } \begin{aligned}
& n=p_{1}{ }^{a} \ldots p_{t}{ }^{a} \text { then } \\
& \text { (2) } \quad a_{k}(n)=\left\{\begin{array}{cl}
\frac{k !}{a_{1} ! \ldots a_{t} !} & \text { if } \Omega(n)=k, \\
0 & \text { otherwise, }
\end{array}\right.
\end{aligned}
$$

With possible applications to the Rlemann zeta function in mind, Ramacbandra bas wabted to know the asympiotic size of the quantity

$$
\max _{k}\left(\sum_{n<N} \frac{a_{k}(n)^{2}}{n^{2 \sigma}}\right)^{\frac{1}{2 k}}
$$

as a function of $\mathrm{N}$ and $\sigma$, where $\sigma$ is fixed, $\frac{1}{2}<\sigma<1$. We settle this question by demonstrating the following

Theorem: Let $\sigma$ be fixed, $\frac{1}{2}<\sigma<1$. With the $a_{k}(a)$ defined by (1), we have

(3) $\max _{k}\left(\sum_{n<N} \frac{a_{k}(n)^{2}}{n^{2 \sigma}}\right)^{\frac{1}{2 k}}=\frac{(\log N)^{1-\sigma}}{\log \log N}$

\footnotetext{
Research supported in part by N S F Grant MCS 80-02559.
} 
In addition, for any integer $k>1$

$$
\left(\sum_{n} \frac{a_{k}(n)^{2}}{n^{2 \sigma}}\right)^{\frac{1}{2 k}} \approx \frac{k^{1-\sigma}}{(\log k)^{\sigma}}
$$

Here the implicit constants may depend only on $\sigma$.

With a little more care one could show that the values of $k$ for which the maximum in (3) is attalned satisfy

$$
k \approx(\log N) / \log \log N \text {. }
$$

To establish that the right band side majorizes the left above we shall require the following two lemmas.

Lemma 1: Let $Y>2$, and put

$$
\mathbf{R}=\mathbf{R}(\mathbf{Y})=\{\mathbf{r}: \mathbf{p} \mid \mathbf{r} \Rightarrow \mathbf{p}<\mathbf{Y}\} \text {. }
$$

Then for any $k>1$, and fixed $\sigma, \frac{1}{2}<\sigma<1$.

$$
\left(\sum_{r \varepsilon R} \frac{a_{k}(r)^{2}}{r^{2 \sigma}}\right)^{\frac{1}{2 k}} \ll \frac{Y^{1-\sigma}}{\log Y}
$$

Proof: Let $X_{2}, X_{3}, \cdots, X_{p}$ be intependent raodom variables, each untformly distributed on the clrcle $|z|=1$. Then

$$
\begin{aligned}
\left(\sum_{r \varepsilon R} \frac{a_{k}(r)^{2}}{r^{2 \sigma}}\right)^{\frac{1}{2 k}} & =\underset{p<y}{\| p_{p}^{\sigma}}\left\|_{2 k}\right\|_{p<y} \frac{X_{p}}{p^{\sigma}} \|_{\infty} \\
& =\sum_{p<y} \frac{1}{p^{\sigma}} \ll \frac{Y^{1-\sigma}}{\log Y} .
\end{aligned}
$$


Lemma 2 : Let $Y>2$, and put

$$
S=S(y)=\{s: p \mid s \Rightarrow p>y\} \text {. }
$$

Then for any $k>1$, and fixed $\sigma, \frac{1}{2}<0<1$.

$$
\left(\sum_{\varepsilon S} \frac{a_{k}(s)^{2}}{s^{2 \sigma}}\right)^{\frac{1}{2 k}} \ll \frac{k^{1 / 2} Y^{\frac{1}{2}}-\sigma}{(\log Y)^{1 / 2}}
$$

Proof : From (2) we see that $a_{k}(s)<k$ ! for all $s$. Thus

$$
\begin{aligned}
& \sum_{i \varepsilon S} \frac{a_{k}(s)^{2}}{s^{2 \sigma}}<k ! \sum_{B \varepsilon S} \frac{a_{k}(s)}{s_{s}^{2 \sigma}}=k !\left(\sum_{p>Y} \frac{1}{p^{2 \sigma}}\right)^{k} \\
& <k !\left(\frac{c Y^{1-2 \sigma}}{\operatorname{lng} Y}\right)^{k} \text {. }
\end{aligned}
$$

and the stated bound follows on taking the $2 k-t h$ root.

We now prove the Theorem. We first show that the left hand side of (3) is at least as largo as the right hand side. Let $Y$ be the largest Integer such that

$$
P=\bigcap_{p<Y} p<N .
$$

Then by the prime number theorem with remainder,

Take $k=\Omega(P)=\pi(Y)$. Theo

$$
Y=\log N+O\left(\frac{\log N}{(\log \log N)^{A}}\right)
$$

$$
\underset{n<N}{a_{k}(n)^{2}} \frac{a_{k}(P)^{2}}{n^{2 \sigma}}>\frac{k !^{2}}{P^{2 \sigma}}>\frac{k !^{2}}{N^{2 \sigma}}
$$

$$
\text { so that }\left(\operatorname{li}_{0 \leqslant N} \frac{a_{k}()^{2}}{n^{2 \sigma}}\right)^{\frac{1}{2 k}} \gg \frac{k}{N^{\sigma / k}} \text {. }
$$


Clearly $k \sim \frac{\log N}{\log \log N}$, and by more careful use of the prime number theorem we see that $k>\frac{\log N}{\log \log N}$ for all large N. Thus $N^{1 / k}<\log N$, and we have the desired lower bousd. Io (4) the value of $k$ is prescribed; we choose $Y$ so that $\pi(Y)=k$, we take $N=P$, and proces as above. We obtain the dealred lower bound since

$$
\frac{(\log N)^{1-\sigma}}{\log \log N} \sim \frac{k^{1-\sigma}}{(\log k)^{\sigma}}
$$

Wo now complete the proof of (3). Let $R$ and $S$ be as in Lemmas 1 and 2. where $Y$ is a parameter to be chosen later. Any $n$ is uniquely of the form $n=r s$ with $r \varepsilon R, s \varepsilon$ S. If $\Omega(D)=k$ and $\Omega(r)=m$ then by (2)

$$
a_{k}(n)=\left(\begin{array}{l}
k \\
m
\end{array}\right) a_{m}(r) a_{k-m}(s) \text {. }
$$

Hence

$$
\sum_{n \leq N} \frac{a_{k}(n)^{2}}{n^{2 \sigma}}=\sum_{m=0}^{k}\left(\begin{array}{l}
k \\
m
\end{array}\right)_{i \varepsilon R}^{2} \sum_{r} \frac{a_{m}(1)^{2}}{r^{2 \sigma}}
$$

$$
\sum_{i s<S} \frac{{ }^{a} k-m(s)^{2}}{s^{2 \sigma}}
$$

Since $\left(\begin{array}{l}k \\ m\end{array}\right)<2^{k}$, this is

$$
\begin{aligned}
& <2^{k} \sum_{m=0}^{k}\left(\begin{array}{l}
k \\
m
\end{array}\right)\left(\underset{r \in R}{\sum_{\varepsilon_{R}} \frac{{ }_{m}(r)^{2}}{r^{2 \sigma}}}\right) \\
& \left(\underset{\substack{s \in S \\
s \in N}}{\sum_{s \in N} \frac{{ }^{a} k-m^{(s)^{2}}}{8^{2 \sigma}}}\right)
\end{aligned}
$$


(5)

$$
<2^{k}\left(R^{2}+8^{2}\right)^{k}
$$

where

$$
R=\max _{m}\left(\sum_{\varepsilon} \frac{{ }_{m}(t)^{2}}{r^{2 c}}\right)^{\frac{1}{2 m}}
$$

and

$$
S=\max _{m}\left(\sum_{s<j}^{\Sigma} \frac{{ }^{\varepsilon} S(s)^{2}}{s^{2 \sigma}}\right)^{\frac{1}{2 m}}
$$

By Lemma 1

$$
\mathrm{R} \ll \frac{\mathrm{Y}^{1-0}}{\log \mathrm{Y}}
$$

To trest $S$ we note that $\Omega(s): m, \& \varepsilon S$, then $s Y^{m}$. If $m>\frac{\log N}{\log X}$ then it follows that $>N$, and the oum defining $S$ will be empty. Thus we may suppose ihat $m<\frac{\log N}{\log Y}$. From thlo bound and Lemma 2 we see that

$$
S \ll \frac{(\log N)^{\frac{1}{2}} Y^{\frac{1}{2}}-0}{\log Y}
$$

On eombining (5), (6), and (7) it follows that

$$
\left(n_{n<N} \frac{a_{k}(0)^{2}}{n^{2 \sigma}}\right)^{\frac{1}{2 k}} \ll \frac{Y^{1-\sigma}}{\log Y}+\frac{(\log N)^{\frac{1}{2}} Y^{\frac{1}{2}-\sigma}}{\log Y} .
$$

and It suffices to take $\mathrm{Y}=\log \mathrm{N}$.

To complete the proof of (4) we may princed more simply. With the $X_{p}$ as in the proof of Lemma ! we see that

$$
\left(\sum_{n} \frac{a_{k}(D)^{2}}{n^{2 \sigma}}\right)^{\frac{1}{2 k}}=\left\|\sum_{p} \frac{X_{p}}{p^{\sigma}}\right\|_{2 k} \text {. }
$$


By the triagle inequality this is

$$
\begin{aligned}
& <\left\|\underset{p<Y}{\sum} \frac{X_{p}}{p_{p}^{\sigma}}\right\| 2 k+\left\|\underset{p>Y Y_{p}^{\sigma}}{\sum} \frac{X_{p}}{p^{\sigma}}\right\| \\
& =\left(\underset{r \varepsilon R}{\sum} \frac{a_{k}(r)^{2}}{2 \sigma}\right)^{\frac{1}{2 k}}+\left(\sum_{s \varepsilon s}^{\sum} \frac{a_{k}(s)}{s^{2 \sigma}}\right)^{\frac{1}{2 k}}
\end{aligned}
$$

By Lemmas 1 and 2 this is

$$
\ll \frac{Y^{1-\sigma}}{\log Y}+\frac{k^{\frac{1}{2}} Y^{\frac{1}{2}}-\sigma}{(\log Y)^{\frac{1}{2}}}
$$

and it suffices to take $Y=k \log k$.

University of Michigan

Ann Arbor, Michigan 48109. 